$16^{\text {th }}$ International Congress of Metrology, 14008 (2013)

DOI: $10.1051 /$ metrology/201314008

(C) Owned by the authors, published by EDP Sciences, 2013

\title{
A high resolution set up devoted to the measurement of the Bidirectional Reflectance Distribution Function around the specular peak, at LNE-CNAM.
}

\author{
Ouarets Shiraz. ${ }^{1}$, Leroux Thierry. ${ }^{2}$, Rougie Bernard. ${ }^{1}$, Razet Annick ${ }^{1}$ and Obein Gaël ${ }^{1}$.
}

1. Laboratoire Commun de Métrologie LNE-CNAM, La Plaine Saint-Denis, France.

2. ELDIM, Hérouville St Clair, France.

Contact shiraz.ouarets@cnam.fr

\begin{abstract}
We see objects because they reflect light. This reflected light contains information that allows us not only identify the shape of the object but also its appearance. The overall appearance of an object is a combination of different visual attributes like gloss, texture, color or transparency, which are produced by the interaction between the object, the light falling upon it and the visual system [1].

Our interest in this article is to characterize the gloss of a surface. Our approach is the measurement of the Bidirectional Reflectance Distribution Function (BRDF) of the surface in, and around the specular direction, where the specular peak is located. For this, we developed a dedicated gonioreflectometer with a very high angular resolution. In this paper we present the equipment and measurements made on different surfaces.
\end{abstract}

\section{Introduction:}

Gloss is defined by the Commission Internationale de l'Eclairage (CIE) to be the mode of appearance by which reflected highlights of objects are perceived as superimposed on the surface due to the directionally selective properties of that surface (2).

Physically, gloss originates from an uneven geometrical distribution of the light reflected by the surface of an object, with an increased flux in the specular direction (3). The reflected light in this direction forms a specular peak whose shape depends strongly on the surface roughness [4], it also depends on the refractive index and of the angle of illumination. According to the surface state we have different types of reflection.

Rough surfaces with not negligible sizes of irregularities foster diffuse reflection with a large and flat peak that gives the sensation of matity [4]. While smooth surfaces reflect most of the light in the specular direction with a sharp peak, that gives the sensation of glossiness.

\section{The BRDF definition}

The physical quantity allowing the description of the specular peak is the Bidirectional Reflectance Distribution Function (BRDF). The BRDF defines the relationship between the radiance reflected in an outgoing direction and the irradiance of the surface in an incoming direction.

BRDF is function of five parameters: and is given by:

$$
\begin{aligned}
\operatorname{BRDF}\left(\theta_{E}, \varphi_{I}, \theta_{R}, \varphi_{R}, \lambda, \Omega\right)= & \\
& \frac{d L_{R}\left(\theta_{E}, \varphi_{I}, \theta_{R}, \varphi_{R}, \lambda \Omega\right)}{d E_{L}\left(\theta_{I}, \varphi_{I}, \lambda\right)}\left(s r^{-1}\right)
\end{aligned}
$$

Where: $\lambda$ is the wavelength of the light $\theta_{\mathrm{I}}$ and $\varphi_{\mathrm{I}}$ are respectively the zenithal and azimuth angles of illumination $\theta_{R}$ and $\varphi_{R}$ are respectively the zenithal and azimuth angles of observation $\Omega$ is the solid angle pointing at the surface, and defined by the aperture of the detection

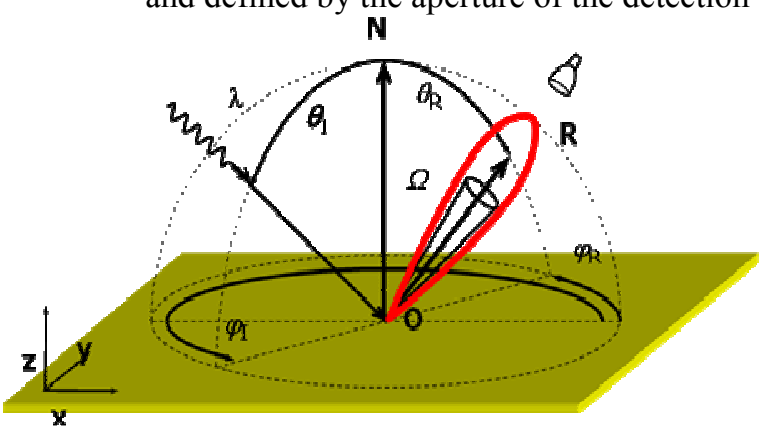

Figure 1. Notation for the BRDF geometry. The red line is an illustration of the specular peak, assuming that the vector $\mathrm{R}$ is in the specular direction.

\section{BRDF and gloss}

A global BRDF describes the angular distribution of the light reflected by the surface of an object in all the half space of observation above the sample. For the 
study of gloss, the measurement of the BRDF can be restricted to the area where the specular peak is located. It means that we will stay in and around the specular direction.

Fresnel assumes that the specular peak height increases as the angle of incidence becomes more grazing. We note that the more the sample's gloss is high, the more the peak height becomes important. This height is also proportional to the refractive index (5)

But previous studies have shown that the height of the peak is not taken into account when the visual system analyses the surfaces gloss (2). We assume that the visual system is more sensitive to the shape of the peak. Thus a good understanding of how the visual system perceive the gloss of a surface requires a good measurement of the shape of the specular peak of that surface. Unfortunately, according to the nature of the sample, the full width at half maximum (FWHM) varies from $15^{\circ}$ for mat surfaces to about $1^{\circ}$ for high glossy surfaces, maybe less.

The measurement of a specular peak with a FWHM of $1^{\circ}$ has never been done but is required for high gloss surface study (figure 2).

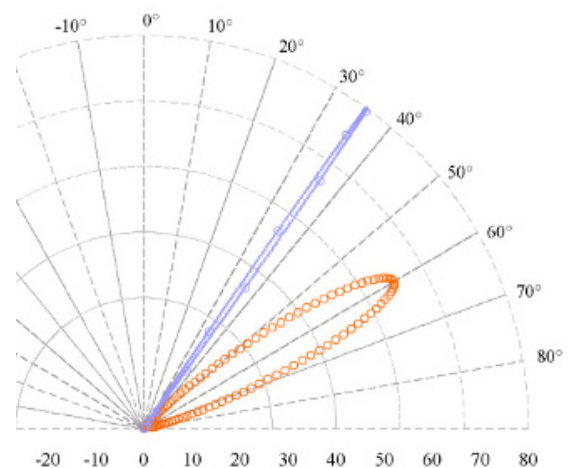

Figure 2. Simulation to illustrate the importance of the angular resolution for specular peak measurements. Purple: Specular peak of a glossy surface with a FWHM of $1^{\circ}$. Orange: specular peak of a matt surface with a FWHM of $10^{\circ}$. Circles: measurement points with an angular resolution of $0,15^{\circ}$. Crosses (only on purple): measurement points with an angular resolution of $0,01^{\circ}$. Only a high resolution can resolve the purple peak.

\section{Measurement bench description}

Our detection is based on the combination of a Fourier optic and a cooled CCD detector to produce the topography of the angular field of the reflected light (6). A target resolution of $0.03^{\circ}$ was chosen because it corresponds to the maximum resolution of the human eye. In the future the result obtained with the instrument will be compared to results obtained by visual evaluations of gloss.

The set up is made of an illumination part, a detection part and a sample holder, placed in a room with grey walls and black curtains to minimize the stray light.
The illumination is made of a broadband source 250$900 \mathrm{~nm}$ placed at twice the focal of a first lens. A pinhole of $200 \mu \mathrm{m}$ of diameter is placed on the source image. This pinhole placed at the focal point of a second collimating lens limits the divergence of the beam at $0.03^{\circ}$. A diaphragm placed after the collimating lens provides a well-collimated $20 \mathrm{~mm}$ diameter beam shown in figure 3

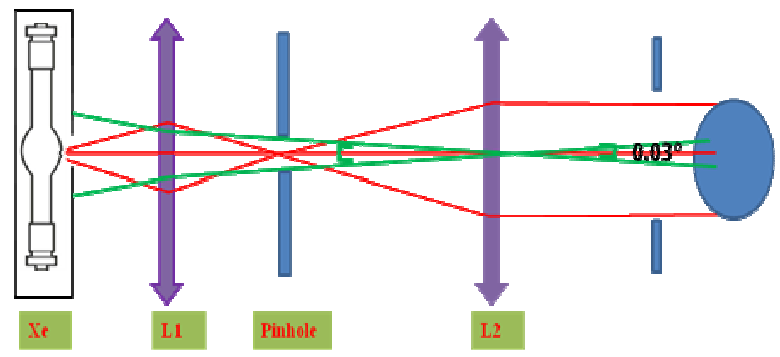

Figure 3. Illumination part that allows to produce a collimated beam with a divergence better than $0,03^{\circ}$.

The detection part combines a Fourier optic and a cooled CCD sensor and has been developed in collaboration with ELDIM $^{2}$. The lens provides a Fourier transform image of the reflected beam coming from the material. Every light beam reflected from the test area with a $\theta_{\mathbf{R}}$ exiting angle is focused on the focal plane at the same azimuth and at a position $x_{i}{ }^{\prime}=\mathrm{f}\left(\theta_{\mathbf{R}}\right)$. $x_{i}$ ' is then reported on the CCD (xi).

Figure 4 shows the principle of the detection.

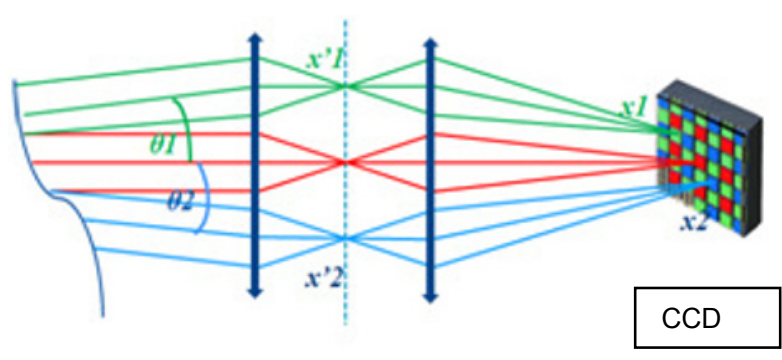

Figure 4. Principle of detection.

In one shoot, the distribution of the luminance reflected in all directions of observation can be measured within a field where $\theta_{\mathbf{R}}<1^{\circ}$.

\section{Characterization of the instrument}

\section{Alignment}

The CCD is not connected to the conoscope, the position between the two instruments is adjustable over a $20 \mathrm{~mm}$ range. The first step to adjust the detection instrument is to find the position of the CCD. 


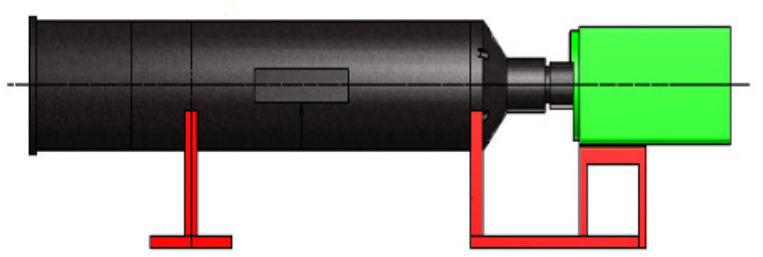

Figure 5. Connection between the camera (green) and the conoscope (black).

The conoscope is designed to focus the parallel rays reflected by the sample in a given direction on the CCD, when the sample is at a distance of $1700 \mathrm{~mm}$. The alignment has been done with a mirror as the sample, and by moving the CCD on the optical axis. We observe the full width at half maximum as a function of the distance between the camera and the conoscope. The smallest peak width corresponds to the best place of the camera.

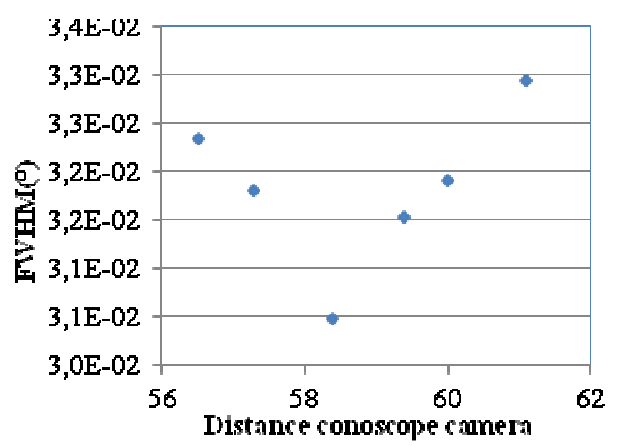

Figure 6. Evolution of the FWHM of the peak obtained with a mirror as the sample according to the distance between the camera and the conoscope. The distance retained is 58,44 $\pm 0.09 \mathrm{~mm}$

\section{Resolution}

Figure 7 shows a cut of the specular peak measurement on a mirror in the plane of incidence. The width of the peak for such a sample is assumed to be $0^{\circ}$. We measured a FWMH equal to $0.03^{\circ}$.

This result is satisfactory. It responds to the divergence of the illumination beam. It confirms that our optical system is capable to reach a resolution of $0.03^{\circ}$.

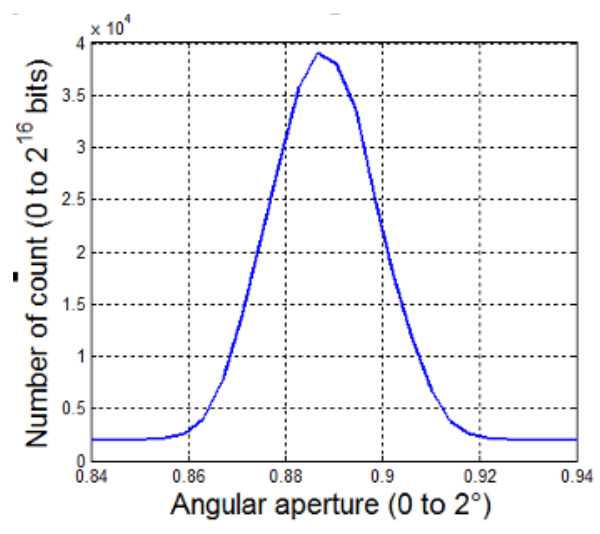

Figure 7. Cut of the specular peak in the plane of incidence for a mirror sample. The full width at half maximum is $0,03^{\circ}$ and depends only of the divergence of the illumination.

The conoscope detects in field of $2^{\circ}$ that corresponds to $512 * 512$ pixel of the CCD. A better resolution could be obtained by reducing the size of the pinhole in the illumination part. We are testing now a pinhole of $75 \mu \mathrm{m}$ of diameter which should give a resolution of $0.01^{\circ}$. The results are encouraging.

\section{The uniformity of the $C C D$}

A CCD is considered uniform when each component returns the same value when exposed to the same light intensity. The sample we use to characterize the CCD uniformity is a Spectralon $\AA$, which is a diffuse reflectance standard from Labsphere. When it is illuminated with a normal incident direction; it reflects light with a near Lambertian behavior. The uniformity of the detection has been tested in this angular configuration. For an exposure time of $60 \mathrm{~s}$ we obtain the topography of the reflected field in all directions as shown in figure 8.

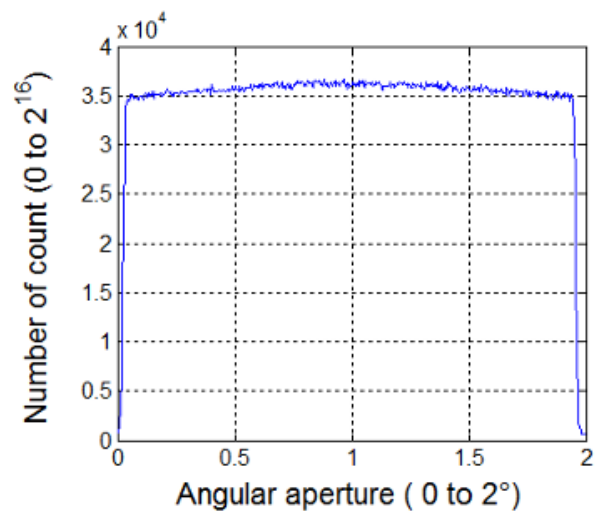

Figure 8. Measurement on a spectralon sample. Incidence at $0^{\circ}$ from the normal. Cut in the plane of incidence of the number of count from 0 to $2^{16}$ bits depending on the angular aperture of the conoscope from $0^{\circ}$ to $2^{\circ}$.

The figure shows that CCD is not uniform, the resulting curve should be flat since each pixel of the CCD is exposed to the same radiance. What we see is 
that the curve decreases sharply on the four CCD sides. A matrix of correction has been computed to compensate this non-uniformity.

\section{Luminous sensitivity}

The luminance of the spectralon has been measured using a spectroradiometer (KONIKA MINOLTA CS2000) with a solid angle of $1^{\circ}$.

A sensitivity matrix has been computed by dividing the luminance measured with the spectroradiometer by each pixel number of count value from 0 to $2^{16}$ bits obtained at a given exposure time $t_{\exp }=60 \mathrm{~s}$. This matrix represents the sensitivity of the detector at a given exposure time.

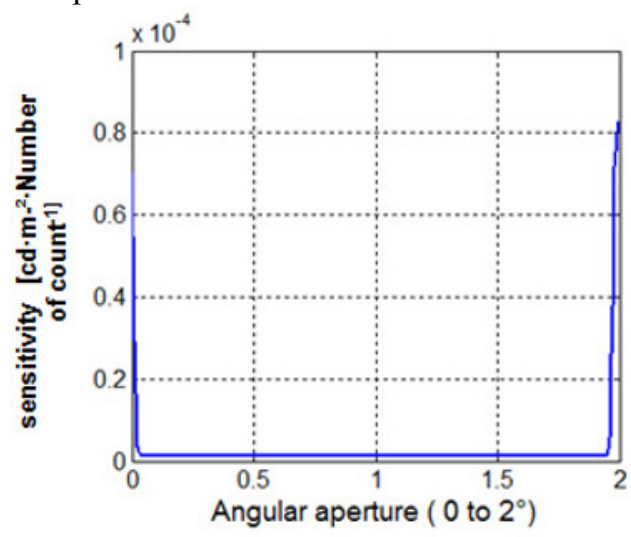

Figure 9. Sensitivity $\left[\mathrm{cd} \cdot \mathrm{m}^{-2} \cdot\right.$ Number of count ${ }^{-1]}$ function of angular aperture of the conoscope from $0^{\circ}$ to $2^{\circ}$.

In order to find the matrix of correction for any exposure time we characterize the temporal sensitivity of the detector.

Measurements of the CCD sensitivity at different exposure time are done. Ideally, the resulting signal should be proportional to the exposure time. Figure 10 shows the results obtained:

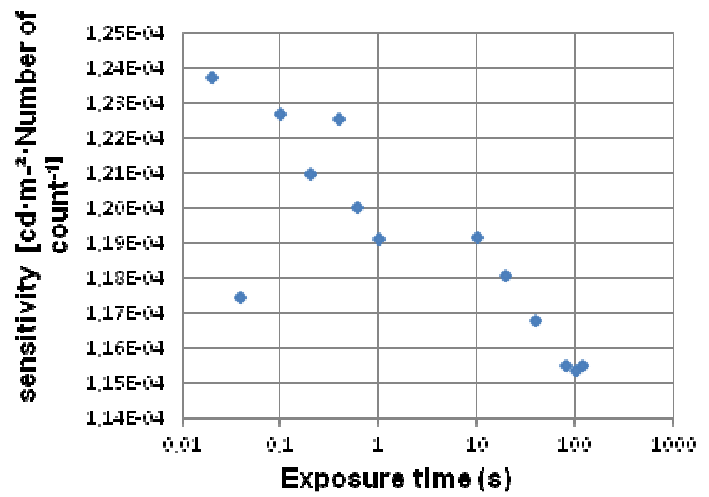

Figure 10. Example of the sensitivity of pixels $\left[\mathrm{cd} \cdot \mathrm{m}^{-2} \cdot\right.$ Number of count $\left.{ }^{-1}\right]$ function of the exposure time

(s). The sensitivity depends of the exposure time and a correction will be applied and is under study.

We see that for short exposure times, the CCD sensitivity is not linear. It means that the correcting matrixes for different exposure times are also not linear for short exposure time. One explanation is that difference between exposure time asked and the real exposure time limited by the shutter performance. The next step will be to correct the exposure time to have a temporal sensitivity more linear.

\section{Conclusion}

The aim of this work was to conceive a detection that can carry on BRDF measurement around the specular direction, in order to characterize the shape of specular peak of glossy samples. The facility we built has an angular resolution of $0,03^{\circ}$ that equal the best resolution of the human visual system. Such a resolution has never been reached.

Whith such a narrow solid angle, the levels of luminance are very low and rise problems of non linearity that have been presented here are still under study. Once fully metrologically characterized, that detection will be integrated in the primary gonioreflectomer of LNE-CNAM (8) and will participate of the measurement made in the framework of the JRP project XDReflect, carried on in the framework of the EMRP research program 2013-2016..

\section{References}

1. Dr Helen White \& Dr Michael R Pointer., The Measurement of Appearance. $9^{\text {th }}$ Congress of the International Color Association.

2. Vocabulaire International de la CIE, $4^{\text {ième }}$ édition, publication CIE n50(845), Bureau central de la CIE, Paris, 1987.

3. Obein G., KnOblauch K., ViÉnot F., 2004, Difference scaling of gloss: Nonlinearity, binocularity, and constancy. Journal of Vision, 4(9), 711-720.

4. Lionel Simonot; PhD report: Etude experimental ET modélisation de la diffusion de la lumière dans une couche de peinture colorée ET translucide, Application à l'effet visuel des glacis ET des vernis (Annexe1). 2003.

5. Gardner/Sward,. Paint testing manual. Physical and chemical examination of paints, varnishes, lacquers, and colors. 13th Edition. 1972.

6. Leroux, Fast contrast vs viewing angle measurements of LCDs, T. Leroux, Eurodisplay, Strasbourg, 447 (1993)

7. Obein G., PhD report: caractérisation optique et visuelle du brillant.2003.

8. Ouarets, S. Ged, G. Razet, A. Obein, G. «A new gonioreflectometer for the measurement of The bidirectional reflectance distribution function (BRDF).-CNAM. CIE 2012 